\title{
WBSCR14, a putative transcription factor gene deleted in Williams-Beuren syndrome: complete characterisation of the human gene and the mouse ortholog
}

\author{
Oscar de Luis, M Carmen Valero and Luis A Pérez Jurado
}

Servicio de Genética, Hospital Universitario La Paz, Madrid, Spain

Williams-Beuren syndrome (WBS) is a neurodevelopmental disorder affecting several systems caused by a heterozygous deletion in the chromosomal region 7q11.23. A common interval that includes up to $\mathbf{1 7}$ genes reported so far is deleted in the great majority of patients. Elastin haploinsufficiency is responsible for the cardiovascular features, but the specific contribution of other deleted genes to the WBS phenotype remains unknown. We have fully characterised a gene commonly deleted in WBS, WBSCR14, previously reported in a truncated form as WS-bHLH. The WBSCR14 CDNA encodes an 852 amino acid protein with a basic helix-loop-helix-leucine-zipper motif (bHLHZip) and a bipartite nuclear localisation signal (BNLS), suggesting a function as a transcription factor. WBSCR14 is expressed as a $4.2 \mathrm{~kb}$ transcript predominantly in adult liver and at late stages of foetal development. The WBSCR14 locus encompasses $33 \mathrm{~kb}$ of genomic DNA with 17 exons. Two intragenic polymorphic dinucleotide repeats have been identified and used to verify hemizygosity in WBS patients. We have also cloned the mouse ortholog and mapped its locus to mouse chromosome 5, in a region of conserved synteny with human 7q11.23. Given that other bHLHZip proteins are dosage sensitive and based on the putative function of WBSCR14 as a transcription factor, hemizygosity at this locus could be involved in some features of WBS. European Journal of Human Genetics (2000) 8, 215-222.

Keywords: Williams-Beuren syndrome; 7q11.23 deletion; bHLHZip domain; transcription factor; hemizygosity

\section{Introduction}

Williams-Beuren syndrome (WBS) is a neurodevelopmental disorder characterised by mental retardation with unique cognitive and personality profiles, distinctive facial features, supravalvular aortic stenosis (SVAS), transient infantile hypercalcaemia, short stature and connective tissue anomalies. ${ }^{1}$ Molecular basis of the syndrome is a heterozygous microdeletion about $1.5 \mathrm{Mb}$ at chromosome band 7q11.23, which has been reported for the great majority of patients studied..$^{2-4}$ Meiotic mispairing of blocks of duplicated sequences that flank the deleted interval followed by unequal inter- or intrachromosomal crossing-over appear to be the

Correspondence: LA Pérez Jurado, Servicio de Genética, Hospital Universitario La Paz, Paseo de la Castellana 261, 28046 Madrid, Spain. Tel: +34 91 3975406; Fax: +34 91 5854587; E-mail: luiz.perez@uam.es Received 26 August 1999; revised 5 November 1999; accepted 22 November 1999 common mechanisms that lead to deletions of similar size in most WBSpatients. ${ }^{5-7}$ The identification of all genes included in the deleted interval, especially those whose haploinsufficiency causes each of the different aspects of the WBS phenotype, is a primary goal when studying WBS. Elastin was the first gene found to be deleted in WBS and its haploinsufficiency has been clearly implicated in SVAS and some of the connective tissue pathology. ${ }^{2,8}$ In addition, deletion of the gene encoding LIM-kinase 1 (LIMK1) has been proposed as a contributing factor to impaired visuo-spatial constructive cognition in WBS, ${ }^{9}$ although this claim was not confirmed by clinical and molecular studies of three different patients with heterozygous LIMK1 deletions. ${ }^{10}$ A total of 15 additional genes has been reported to be part of the common WBS deleted region although none of them has been related to any of the remaining phenotypic features of the WBS phenotype (see Francke ${ }^{11}$ for a review). They include the 
single copy genes coding for the immunophilin FK-506 binding protein FKBP6, a D rosophila frizzled homolog (FZD9), the putative transcription factor WBSCR9 also known as WSTF, the homolog of a gene disrupted by a leukemic translocation breakpoint (BCL7B), a $\beta$-transducin (TBL2 also named $\mathrm{WS}-\beta \mathrm{TRP})$, the putative transcription factor WS-bHLH, the presynaptic vesicle protein syntaxin $1 A$ (STX1A), the Clostridium perfringens enterotoxin receptors 1 and 2 (approved symbols CLDN 4 and CLDN3), the eukaryotic initiation factor EIF4H also known as WBSCR1, the subunit 2 of the replication factor C (RFC2), the neuronal cytoplasmatic linker protein CYLN2, and the GTF2l-related protein GFT2IRD1/WBSCR11. The transcription initiator factor GTF2I and the neutrophilic cytosolic factor 1 NCF1 are part of duplicons that flank the WBS deletion, with the functional copy of both genes being at the telomere edge of the deleted interval. ${ }^{6,11}$

Weand others have constructed meiotic and physical maps that totally or partially cover the deleted region. ${ }^{12-15}$ Some of the BAC clones have been sequenced as part of the Human Genome Project and a great portion of the region is al ready sequenced and available in public databases, albeit as unordered pieces. Using this resource, we have fully characterised W BSCR14 (nomenclature committee approved gene symbol), a gene previously reported as WS-bHLH (Genbank acc. no. AF056185). However, we have found significant differences between WBSCR14 and WS-bHLH cDNAs, suggesting that the reported WS-bHLH CDNA was a chimeric and truncated form. We have also identified the mouse ortholog and mapped its locus to a region of conserved synteny with human chromosome7q11.23.

\section{Materials and methods \\ cDNA cloning and genomic structure}

The genomic sequence from BAC RG315H11 (Genbank acc. no.AC005089) was analysed using the integrated NIX resource available at the web pages of the UK Human Gene Mapping Project (http://www.hgmp.mrc.ac.uk). ESTs with identity to predicted exons were identified and assembled by computer analysis. Several oligonucleotide primers were designed and used to obtain a cDNA by reverse transcribed (RT)-PCR from human adult liver total RNA. Sequencing of PCR products was performed using an $A B I 377$ machine (Perkin-Elmer, Roche Molecular Systems Inc, Branch burg, NJ, USA) according to the manufacturer's instructions. Assembling of two non-overlapping sequenced pieces of BAC RG315H11 was done by PCR with primers WS4B: 5'-CAG CCT GGG CAA CAG ATT CA-3'; and 1R: 5'-GTA TTC CCG CAT CAT CAT CT-3'. Comparison of CDNA and genomic sequence permitted the estimation of intron sizes and the identification of intron-exon junctions and boundaries. Transcription initiation was predicted by using the PPNN (http://www.hgc-lbl.gov/projects/promoter.html) and Matl nspector (http://www.genomatix.gsf.de) programs.

\section{Mapping of WBSCR14 and WS-bHLH sequences}

Somatic cell hybrid lines containing a single human chromosome in a rodent background were obtained from the NIGMS Human Genetic Mutant Cell Repository (Coriell Institute for Medical Research, Camden, NJ, USA). PCR screening of DNAs from the panel of hybrid cells was performed with primers specific for the 5' of the W S-bHLH gene: MD: 5'-TCT CAA ACC CTC TCC CTG AC-3'; and MR: 5'-GTC AGT CAT AGC AGC TCA CC-3'. Refined mapping of the W BSCR14 locus among other genes in the region was performed by STS analysis of several cosmids previously identified with a TBL2 probe. $^{16}$

\section{Patients and polymorphism genotyping}

Criteria for clinical diagnosis of WBS have been previously reported. ${ }^{3}$ Fourteen patients with a complete genetic evaluation who fulfil diagnostic criteria were studied. Genomic DNA was obtained from each patient and both parents by using the Puregene DNA Isolation Kit (Gentra Systems, Minneapolis, USA). Fluorescent-labelled oligonucleotides were designed for amplification of the polimorfic (CA)n markers D7S2476 and WS4. Sequences of the oligonucleotides designed for WS4 were WS4B, described above, and WS4A: 5'-CCA AAG CAC TGG GAT TAC AG-3'. PCR reactions were performed in a final volume of $10 \mu \mathrm{l}$, using standard conditions. PCR products were resolved using an $A B I$ Prism 310 genotyper. Additional molecular diagnosis of patients was made by the analysis of other intradeletion polymorphic markers previously reported along with FISH in some cases. ${ }^{3}$

\section{Expression analysis}

Northern blot and dot blot filters containing poly $(\mathrm{A})^{+} \mathrm{mRNA}$ from multiple human and mouse tissues were purchased from Clontech (Clontech Laboratories, Palo Alto, CA, USA) and probed with human and mouse CDNA probes according to the manufacturer's instructions. Probes were generated by RT-PCR from adult liver total RNA or total RNA of whole mouse embryo, respectively, using the same primers in both cases: 6D: 5'-GAG AAC CGA CGT ATC ACA CA-3'; and UR: 5'-CTC TGT GAC TGC CCG TGT GG-3'. The probes were gel purified and radiolabelled using a random priming labelling kit (Amersham Pharmacia Biotech, Uppsala, Sweden). Hybridisation was performed at $65^{\circ} \mathrm{C}$ for $16 \mathrm{~h}$, and filters were washed at a final stringency of $0.1 \mathrm{XSSC}, 0.1 \% \mathrm{SDS}$ at $65^{\circ} \mathrm{C}$. As a control for RNA loading and quality, filters were stripped and rehybridised using a $\beta$-actin probe.

\section{Cloning and mapping of the mouse Wbscr14 gene}

ESTs with homology to the human cDNA were searched using the BLAST algorithm. ${ }^{17}$ The Wbscr14 CDNA was obtained by RT-PCR combining degenerate primers designed from the human sequence and mouse specific primers designed from ESTs. The yeast artificial chromosome (YAC) WI/MIT library no. 820 was previously screened with several 
CDNA probes from the genes deleted in WBS. ${ }^{18}$ DNA from the positive YAC clones was analysed by $P C R$ with mouse W bscr14 primers 9D: 5'-ACC TCA CTG GCC TGG CTG GA-3' and UR (above). For long-range restriction mapping, high molecular weight mouse genomic DNA was digested with restriction endonucleases $\mathrm{Mlul}$ and $\mathrm{Notl}$, blotted on to nylon membranes and hybridised with W bscr14, Stxla and Tbl2 specific probes. DNA radioactive labelling and hybridisation were performed as described for the northern blot assays.

\section{Results and discussion}

Computer analysis of genomic sequence from BAC RG315H11 using the integrated NIX resource allowed the identification of several overlapping ESTs with identity to predicted exons. ${ }^{17}$ Additional putative coding exons were identified by conserved homology across species with a Caernorhabditis el egans open reading frame (ORF) (T20B12.6) predicted from genomic sequencing. ${ }^{19}$ A W BSCR14 CDNA was obtained by assembling overlapping RT-PCR products from adult liver total RNA. The obtained $3270 \mathrm{nt}$ W BSCR14 CDNA contains a $2559 \mathrm{nt}$ ORF that encodes an 852 amino acid protein, with a predicted molecular weight of $93.1 \mathrm{kDa}$. The putative translation initiation has a good Kozak consensus. ${ }^{20}$ A splicing variant due to the use of an alternative acceptor splice site at the beginning of exon 11 results in a trinucleotide (CAG) deletion in the mRNA (Table1) and the corresponding del etion of a glutamine residue at position 558 of the protein. No additional splicing variants were identified in the WBSCR14 CDNA by RT-PCR with combinations of primers.

The predicted WBSCR14 protein contains three known functional motifs: a bipartite nuclear localisation signal (BNLS; aa 158-175), ${ }^{21}$ a basic helix-loop-helix (bHLH) region $(\text { aa 650-704) })^{22}$ and a consensus leucine-zipper (Zip) domain $L(X)_{6} L(X)_{6} L$ (aa 704-725) (Figure $\left.1 A\right) .{ }^{23}$ Stretches of prolinerich sequences (aa382-471 and 571-585) as well as addi- tional non-consensus Zip-like motifs (aa725-738 and 795-835), were also detected. Several regions of the protein including the BNLS and bHLH regions are quite conserved (45-51\% similarity) in the Caenorhabditis elegans protein T20B12.6 (Figure1A).

The previously reported $2665 \mathrm{nt}^{\mathrm{W}} \mathrm{S}-\mathrm{bHLH} \mathrm{CDNA}^{12}$ is identical to WBSCR14 CDNA at the $3^{\prime}$ end, in a region corresponding to exons 9,10 and from 12 to 17 of the W BSCR14 genomic structure (Figures $1 B$ and 2). However, the initial 918 nt of W S-bHLH cDNA were not found in the cloned W BSCR14 CDNA nor in the genomic sequence of BAC RG315H11. PCR with primers designed from this sequence (MD and MR) amplified the expected $134 \mathrm{bp}$ fragment from control DNA as well as from a cell line containing a single human chromosome7 with a typical WBS interstitial deletion in a hamster background, ${ }^{24}$ indicating the location of at least one copy outside the common deletion (not shown). Using the same primer pair on a somatic cell hybrid panel in which each cell line contains a single human chromosome in a rodent background (Coriell Repositories), specific amplification products were obtained from human chromosomes 3 and 13 along with human genomic DNA; weak amplification was detected from chromosome 21 , and no product from any other human chromosome (including chromosome7), mouse and hamster genomic DNA. In addition, PCR with MD and several WBSCR14 CDNA reverse primers failed to obtain any amplification product on reverse transcribed adult human liver mRNA. Therefore, we believe that the reported W S-bHLH CDNA was probably a chimeric fragment due to a cloning artefact. W S-bHLH coded for a putative protein of 231 residues, almost identical to the carboxyterminus of WBSCR14 except for the absence of two residues in WS-bHLH due to the deletion of $6 \mathrm{nt}$ at the beginning of exon 13 (Figure1A). The predicted WS-bHLH protein contained the bHLH and Zip domains but lacked the proline-rich segments and the BNLS.

Table 1 Exon/intron organization of human WBSCR14 gene

\begin{tabular}{|c|c|c|c|c|c|}
\hline Exon & (bp) 3' Intron & 5' Exon & $\begin{array}{l}\text { Length } \\
\text { (bp) }\end{array}$ & 3' Exon & $5^{\prime}$ Intron (bp) \\
\hline 1 & & CCCCGC.... & 340 & ....CTACAG & gtgagggc...(8023) \\
\hline 2 & (8023)...ctcccctttgacag & TGGCAA.... & 106 & ....TCCAGT & gtgagtgg...(8353) \\
\hline 3 & (8353) ...cccccttctctcag & ATGTGA.... & 83 & ....CGGAGG & gtagctgg.....(169) \\
\hline 4 & (169) ...ggcccctaccccag & CCGTGG.... & 88 & ....AAGCGG & gtcagtgg.....(311) \\
\hline 5 & (311)...gtgtgtccctgcag & СTCCGT.... & 44 & ....AAGCAG & gtgggtgc.....(864) \\
\hline 6 & (864)...ctctgtctccctag & GCGGAA.... & 201 & ....AGGATG & gtgagggt.....(142) \\
\hline 7 & (142)...actgctgcctccag & CСTACG.... & 80 & ....TСТСAG & gtggggcg...(5913) \\
\hline 8 & (5913)...tgtctgaattacag & АТТТСТ.... & 169 & ....CTGCAG & gtgagctc...(1813) \\
\hline 9 & (1813)...gtccccccaaccag & GCTCGG.... & 531 & ....CAGCAG & gtgagggc.....(250) \\
\hline 10 & (250)...ctgcccgtgcacag & СTAAGC.... & 67 & ....TCCCCG & gtaagatc..(70/73) \\
\hline 11 & (70/73) ctccaccccag (cag) & ( CAG ) GAG & $153 / 150$ & ....CCAGCG & gtaaagag.....(160) \\
\hline 12 & (163)...tgtccctaccacag & GCAGTG.... & 115 & ....AACAAG & gtgggcac......(92) \\
\hline 13 & (92) ...ctctgctccccgag & ACCGAG.... & 119 & ....CTCAAG & gtgagccc.....(184) \\
\hline 14 & (184)...ggtggcgtctgcag & GTGAGC.... & 127 & ....САТТАА & gtaggcag.......(82) \\
\hline 15 & (82) ...tgccctgсcсcсag & CCTGTG.... & 123 & ....TGGGTG & gtatcctc...(1234) \\
\hline 16 & (1234)...tgacctgggcccag & TTCAGC.... & 129 & ....GGCCAA & gtacgtga.....(291) \\
\hline
\end{tabular}




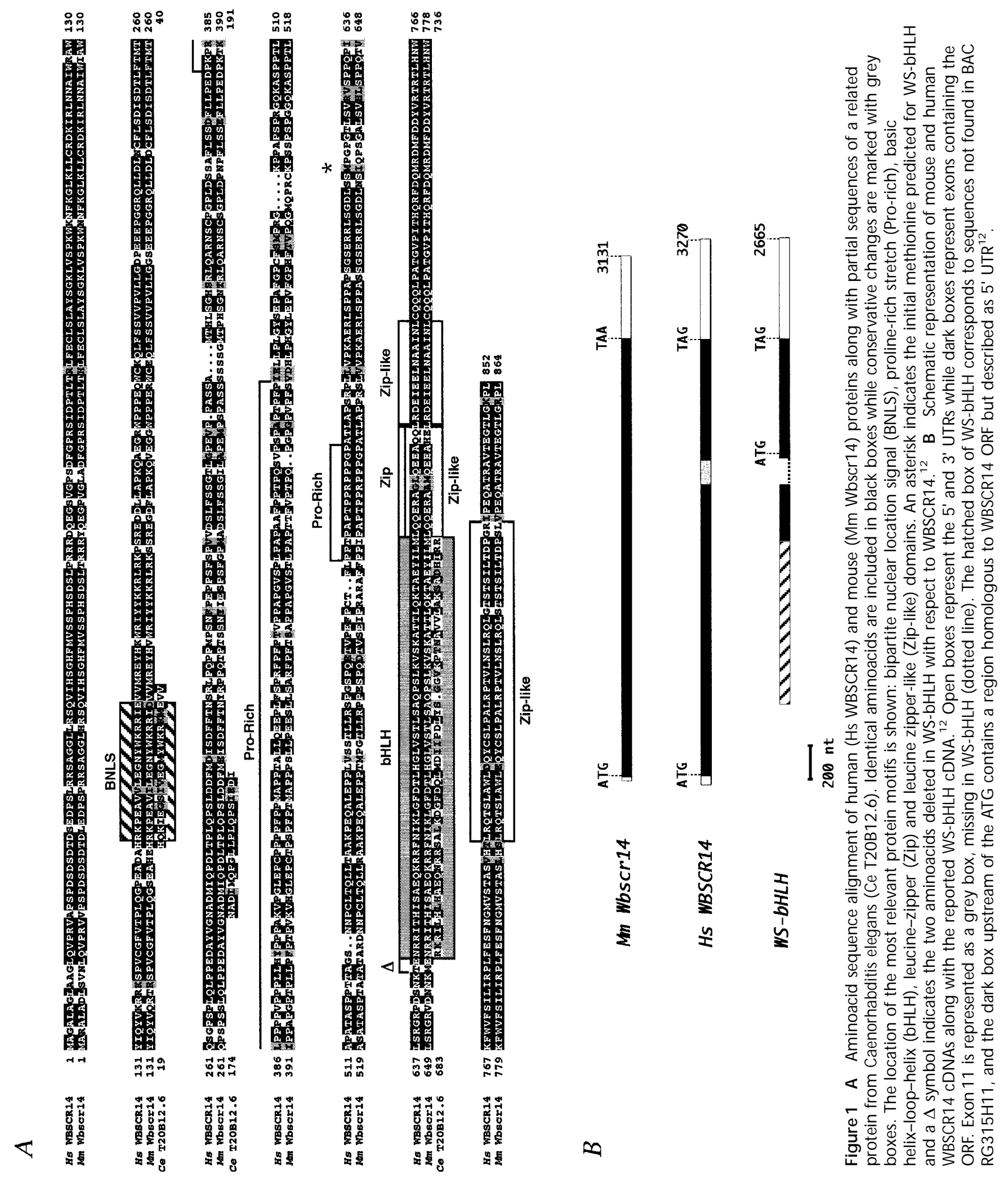




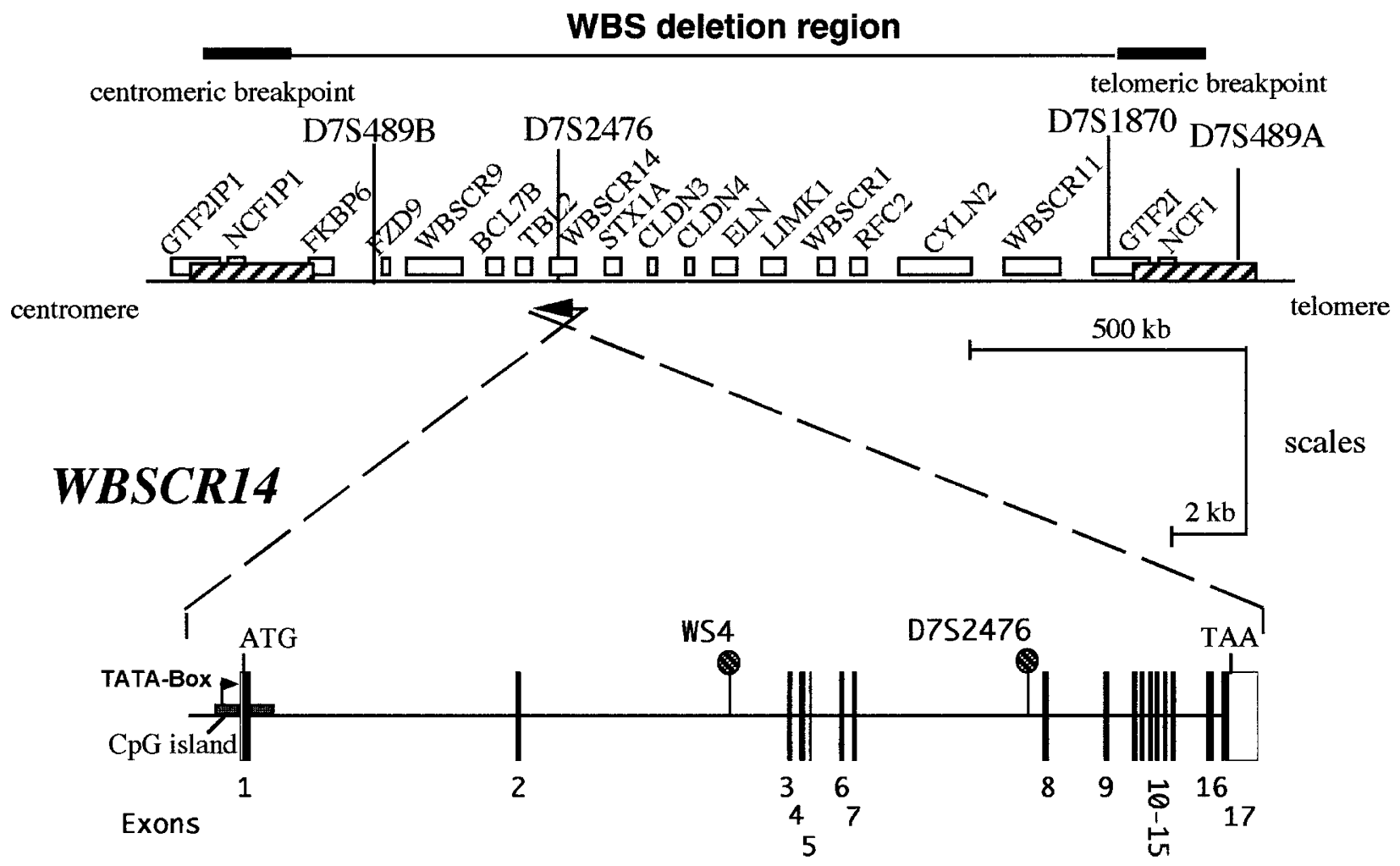

Figure 2 Mapping and genomic structure of the WBSCR14 gene. Top: Schematic representation of the transcription map of the WBS commonly deleted region in $7 q 11.23,{ }^{11}$ with the location and transcriptional orientation (indicated by an arrow) of the WBSCR14 gene. Bottom: Genomic structure of the WBSCR14 gene with the 17 exons represented by boxes numbered below. Open box contains 3' untranslated region (UTR). The locations of the CpG island with the predicted TATA box and the two intragenic CA repeats, WS4 and D7S2476, are indicated.

PCR with primers WS4B and $1 R$ on genomic DNA obtained the expected $2.2 \mathrm{~kb}$ fragment allowing the joining of two non-overlapping sequenced pieces of BAC RG315H11 and the completion of the W BSCR14 genomic sequence (Genbank acc. no. AF156673). The exon-intron structure of W BSCR14 was determined by comparison of W BSCR14 CDNA and genomic sequences. The gene encompasses $33 \mathrm{~Kb}$ of genomic DNA, including 17 exons with 16introns (Figure2). All intron-exon junctions conform to the AG-GT rules (Table1). A CpG island surrounds the first exon and a good putative transcription initiation site with a TATA box $23 \mathrm{bp}$ upstream was predicted. BAC RG315H11 also contains other genes included in the WBS critical region such as TBL2/ W S- $\beta$ TRP and BCL7B. ${ }^{12,16}$ To determine the transcriptional orientation of the WBSCR14 gene and its rel ative order with respect to the other genes in the region, PCR analysis with several STSs developed from the genomic sequence was performed using overlapping cosmids previously obtained with a TBL2 probe. $^{16}$ The results were consistent with the order depicted in Figure2, all three genes being in the same transcriptional direction. Our results agree with two recent reports $^{13,14}$ but differ from others. ${ }^{12,15}$

The location of WBSCR14 within the 7q11.23 WBS deletion is strongly supported by our mapping studies along with the previous definition of the commonly deleted interval (Figure2). ${ }^{3,4}$ However, for additional confirmation, we used two WBSCR14 intragenic polymorphic $(C A)_{n}$ repeats to determine allelic inheritance in WBS individuals. One of them corresponds to the previously described locus D7S2476, which was found in intron 7 of the gene and has a heterozygosity of $70 \%$. The second marker, named WS4, was identified in intron 2 of the gene and typed using specific primers WS4A and WS4B. The observed heterozygosity of WS4 in 28 unrelated individuals was $40 \%$, with four different alleles found. Lack of parental inheritance at both loci was observed in all informative WBS patients genotyped, corroborating the location of WBSCR14 within the 7q11.23 common deletion (data not shown).

To obtain the mouse orthologous gene, several mouse ESTs with homology to the human CDNA were identified through a BLAST search. ${ }^{17}$ A 3131 nt mouse W bscr14 cDNA was obtained by RT-PCR combining degenerate primers designed from the human sequence and mouse specific primers designed from ESTs. It contains a $2595 \mathrm{nt}$ ORF that codes for a 864 amino acid protein with a predicted molecular weight of $94.8 \mathrm{kDa}$. No alternative splicing was found in the mouse CDNA sequence. Human and mouse protein sequences display $81 \%$ identity and $85 \%$ similarity, the homology being 
A

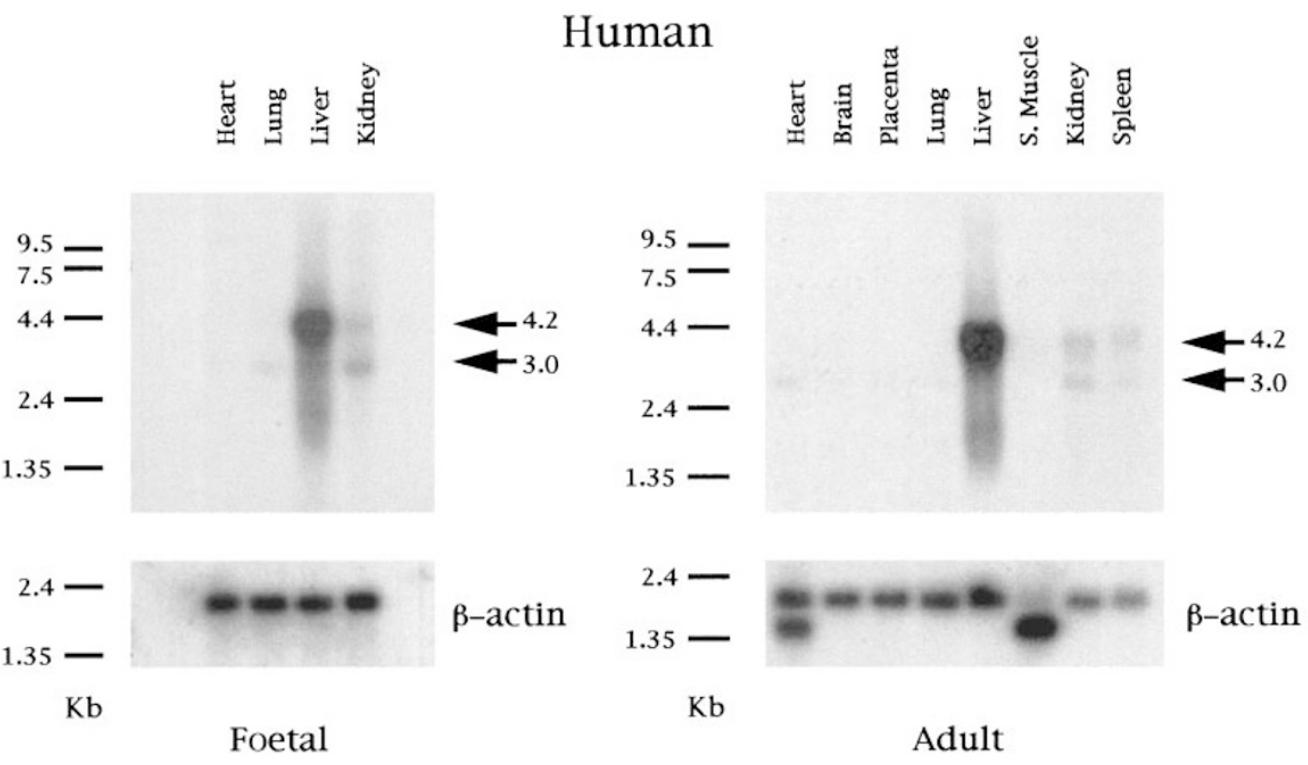

B

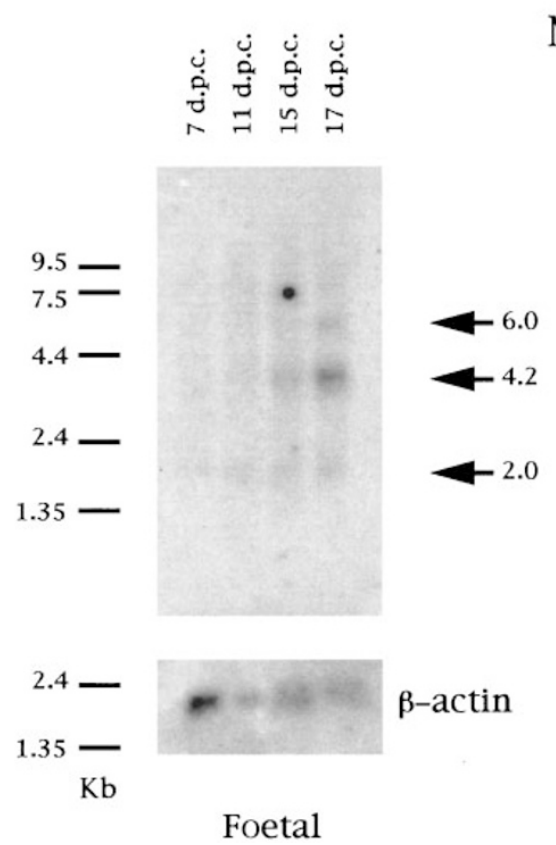

Mouse
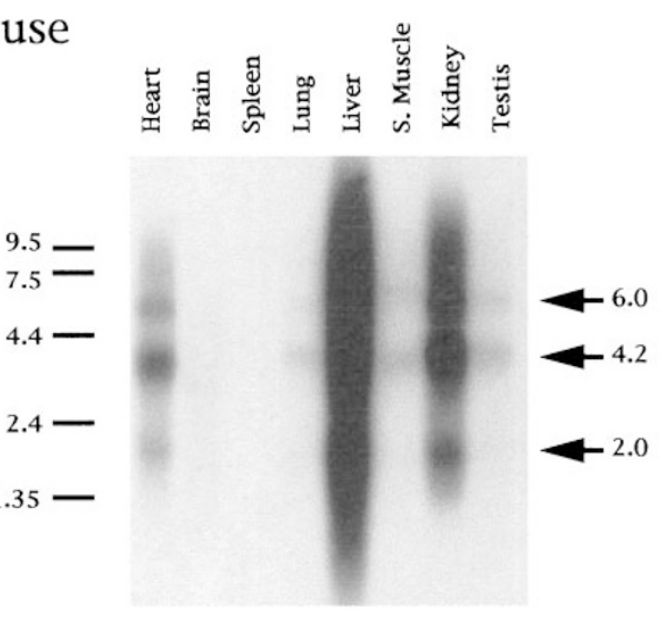

$$
2.4-
$$

$\mathrm{Kb}$

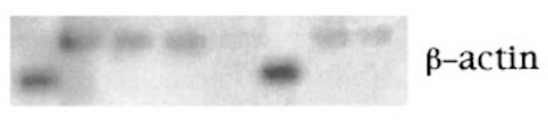

Adult

Figure 3 Expression analyses of human A and mouse B WBSCR14 genes in foetal and adult tissues. Tissues used are described on top of each lane. dpc: days of postcoitum mouse foetal development. A A major transcript of $4.2 \mathrm{~kb}$ is observed in adult and foetal liver in humans with almost no signal in other tissues. B In mouse, a similar band of $4.2 \mathrm{~kb}$ is found in adult liver with weaker signal in kidney and heart. During development, the $4.2 \mathrm{~kb}$ Wbscr14 transcript is barely detected at $15 \mathrm{dpc}$ and increases thereafter. The hybridisation of the $\beta$-actin probe used as control for RNA loading and quality is seen below each blot. 
higher at the amino- and carboxy-terminal regions where the main putative functional domains are located (Figure1A).

The W bscr14 locus was mapped to mouse chromosome 5 by physical methods. We had previously obtained overlapping yeast artificial chromosomes (YACS) covering the mouse region that contains the orthologs to genes del eted in WBS, from Fkbp6 to N cf1. ${ }^{18}$ By PCR and hybridisation, $W$ bscr14 was found in two overlapping YACs of the region (WI/MIT library YAC addresses 446D3 and 403H9). In addition, long range restriction mapping by pulse-field gel electrophoresis revealed that the W bscr14 locus is located between Tbl2 and Stxla (not shown). These mapping results provide an additional proof that we have identified the true ortholog of WBSCR14, since they are consistent with the conservation of synteny between mouse chromosome 5 band $\mathrm{G} 1$ and human chromosome 7q11.23. ${ }^{18}$

Northern blots from multiple tissues hybridised with a W BSCR14 specific proberevealed a $4.2 \mathrm{~kb}$ si gnal detected very predominantly in liver and significantly weaker in kidney (Figure $3 \mathrm{~A}$ ). With longer exposure, the $4.2 \mathrm{~kb}$ band as well as an additional transcript of $3.0 \mathrm{~kb}$ was detected in most tissues. The same pattern was obtained in foetal tissues with strong signal in liver, much weaker in kidney and barely detectable in heart and lung (Figure $3 \mathrm{~A}$ ). Our results are quite similar to those previously reported with a W S-bHLH probe. A dot-blot was also used to obtain a broader and quantitative spectrum of WBSCR14 expression. A strong signal was evident in adult liver and much weaker in foetal liver, with almost no detectable signal in any other tissue (not shown). Expression pattern of the mouse gene was obtained with a mouse cDNA probe. In adult animal tissues, a main transcript of $4.2 \mathrm{~kb}$ was observed in liver, weaker in kidney and heart, with additional weak bands of 2.0 and $6.0 \mathrm{~kb}$ with proportional intensities (Figure 3B). A strong smear was also seen in adult liver and we believe that this was likely due to the presence of RNA aggregates and/or RNA degradation in the sample of that specific lane. On a developmental northern blot, W bscr14 expression was barely detectable at $15 \mathrm{dpc}$ of foetal development, increasing significantly by $17 \mathrm{dpc}$ (Figure3B).

Basic helix-loop-helix (bHLH) and basic leucine-zipper (bZip) transcription factors have highly conserved bipartite domains for protein-protein (HLH or Zip motifs) and DNAprotein (basic region) interaction; they act as important regulators during processes of cell proliferation and differentiation. ${ }^{22,23,25}$ M ost of them are expressed during development in a tissue- and stage-specific pattern. Examples of bHLH transcription factors include MyoD and NeuroD, which regulate the tissue-specific gene expression that controls cell determination and differentiation in myogenesis and neurogenesis, respectively. ${ }^{26,27}$ The presence of both, bHLH and Zip regions in some proteins, is thought to enhance protein dimerisation capabilities. The bHLHZip domain specifies dimerisation and determines sequence specific DNA binding at the E box (CANNTG) consensus on the promoter of target genes. ${ }^{12}$ Haploinsufficiency causing human genetic diseases has been shown at genes coding for bHLH proteins. The MITF gene encodes a bHLHZip transcription factor that plays a key role in melanocyte differentiation. ${ }^{28}$ Null mutations at one allele of the MIFT locus result in the Waadenburg syndrome type $2 \mathrm{~A}$, a disease that associates neurosensorial deafness with pigmentary disturbances. $^{29}$ The TW IST gene encodes another bHLH transcription factor proposed to function as an upstream regulator of fibroblast growth factor receptor genes. Haploinsufficiency for TWIST causes the autosomal dominant disorder SaethreChotzen syndrome, a relatively common form of craniosynostosis. $^{30}$

The task of determining whether any of the deleted genes in WBS contributes to particular features of the phenotype is difficult. To date, clinical-molecular correlations in some exceptional patients with partial deletions have suggested that the genes mainly responsible for abnormal cognition map to the telomeric interval of the deletion, whilst genes from FKBP6 to W BSCR14, at the centromeric edge of the deletion, might contribute to other phenotypic aspects including mental retardation. ${ }^{10}$ However, these conclusions lack strong supportive evidence and additional clinical and molecular studies are required. W BSCR14 is included in the common interval deleted in WBS patients. Like other genes encoding bHLH-type transcription factors, W BSCR14 may be dosage sensitive and its heterozygous deletion could have phenotypic consequences.

Participation of bHLH and bZip transcription factors in vertebrate development suggests that other family members such as WBSCR14 can also be implicated in developmental patterns that, when altered by insufficient dosage, may lead to morphological and/or functional abnormalities. The expression pattern of W BSCR14 suggests a main involvement in liver regulatory functions and during late embryonic development although mRNA expression is not always a suitable parameter to predict the function of a gene. A definitive proof of involvement of W BSCR14 in any aspect of the WBS phenotype awaits the identification of patients with specific manifestations of the syndrome and intragenic mutations. Elucidation of the specific cellular function of WBSCR14, identification of its target genes and interacting proteins as well as the inactivation of the gene in mouse, will be helpful to further define its putative implication in WBS.

\section{Acknowledgements}

We thank $\mathrm{Dr}$ J Cruces for his support and critical reading of the manuscript, P Casas and J López Monclús for excellent technical assistance, Dr R Peoples for cell hybrid DNA, and the W BS patients and families for their participation. This work was supported by grants from the FIS (98/1062) and CAM (08.6/0004/98). 


\section{References}

1 Greenberg F: Williams syndrome professional symposium. Am J Med Genet 1991; 6(suppl): 82-88.

2 Ewart AK, Morris CA, Atkinson D et al: Hemizygosity at the elastin locus in a developmental disorder, Williams syndrome. Nat Genet 1993; 5: 11-16.

3 Pérez Jurado LA, Peoples R, Wang Y-K et al: M olecular definition of the chromosome 7 deletion in Williams syndrome and parentof-origin effects on growth. Am J Hum Genet 1996; 59: 618-625.

$4 \mathrm{Wu}$ YQ, Sutton VR, Nickerson E et al: Delineation of the common critical region in Williams syndrome and clinical correlation of growth, heart defects, ethnicity, and parental origin. Am J Med Genet 1998; 78: 82-89.

5 Dutly F, Schinzel A: Unequal interchromosomal rearrangements may result in elastin gene deletions causing the Williams-Beuren syndrome. Hum Mol Genet 1996; 5: 1893-1898.

6 Pérez Jurado LA, Wang Y-K, Peoples R, Coloma A, Cruces J, Francke U: A duplicate gene in the breakpoint regions of the Williams-Beuren syndrome del etion encodes the initiator binding protein TFII-I and BAP-135, a phosphorylation target of BTK. Hum Mol Genet 1998; 7: 325-334.

7 Urbán Z, Helms C, Fekete G et al: 7q11.23 deletions in Williams syndrome arise as a consequence of unequal meiotic crossover. Am J Hum Genet 1996; 59: 958-962.

8 Tassabehji M, Metcalfe K, Donnai D et al: Elastin: genomic structure and point mutations in patients with supravalvular aortic stenosis. Hum Mol Genet 1997; 6: 1029-1036.

9 Frangiskakis JM, Ewart AK, Morris CA et al: LIM-kinase1 hemizygosity implicated in impaired visuospacial cognitive cognition. Cell 1996; 86: 59-69.

10 Tassabehji M, Metcalfe K, Karmiloff-Smith A et al: Williams syndrome: use of chromosomal microdeletions as a tool to dissect cognitive and physical phenotypes. Am J Hum Genet 1999; 64: 118-125.

11 Francke U: Williams-Beuren syndrome: genes and mechanisms. Hum Mol Genet 1999; 8: 1947-1954.

12 Meng X, Lu X, Li Z et al: Complete physical map of the common deletion region and characterization of three novel genes. Hum Genet 1998; 103: 590-599.

13 Osborne LR, Campbell T, Daradich A, Scherer SW, Tsui LC: Identification of a putative transcription factor gene (W BSCR11) that is commonly deleted in Williams-Beuren syndrome. Genomics 1999; 57: 279-284.

14 DeSilva U, Massa H, Trask BJ, Green ED: Comparative mapping of the region of human chromosome 7 deleted in Williams syndrome. Genome Res 1999; 9: 428-436.

15 Hockenhull EL, Carette MJ, Metcalfe K, Donnai D, Read AP, Tassabehji M: A complete physical contig and partial transcript map of the Williams syndrome critical region. Genomics 1999; 58 : 138-145.
16 Pérez Jurado LA, Wang Y-K, Francke U, Cruces J: TBL2, a novel transducin family member in the WBS deletion: characterization of the entire sequence, splice variants and the mouse ortholog. Cytogenet Cell Genet 1999; 86: 277-284.

17 Altschul SF, Madden TL, Schaffer AA et al: Gapped BLAST and PSIBLAST: a new generation of protein database search programs. Nucleic Acids Res 1997; 25: 3389-3402.

18 Valero MC, Cruces], Pérez Jurado LA: Syntenic organization of the Williams-Beuren syndrome deleted region in chromosome7q11.23 and the mouse chromosome5 band C. Am J Hum Genet 1998; 63(suppl.): Abstract no. 1495.

19 Wilson R, Ainscough R, Anderson $\mathrm{K}$ et al: $2.2 \mathrm{Mb}$ of contiguous nucleotide sequence from chromosomelll of $C$. elegans. Nature 1994; 368: 32-38.

20 Kozak M: Point mutations define a sequence flanking the AUG initiator codon that modulates translation by eukaryotic ribosomes. Cell 1996; 44: 283-292.

21 Dingwall C, Laskey RA: Nuclear targeting sequences-a consensus? Trends Biochem Sci 1991; 16: 478-481.

22 Krause M, Fire A, Harrison SW, Priess J, Weintraub H: CeMyoD accumulation defines the body wall muscle cell fate during $C$. elegans embryogenesis. Cell 1990; 63: 907-919.

23 Agre P, Johnson PF, McKnight SL: Cognate DNA binding specificity retained after leucine zipper exchange between GCN4 and C/EBP. Science 1989; 246: 922-926.

24 Peoples RJ, Pérez Jurado LA, Wang Y-K, Kaplan P, Francke U: The gene for the replication factor $C$ subunit 2 (RFC2) is within the 7q11.23 Williams syndrome deletion. Am J Hum Genet 1996; 58: 1370-1373.

25 Sassone-Corsi P: Transcription factors responsive to CAMP. Annu Rev Dev Biol 1995; 11: 355-377.

26 Lee JE: NeuroD and neurogenesis. Dev Neurosci 1997; 19: 27-32.

27 Olson EN, Klein WH: bHLH factors in muscle development: dead lines and commitments, what to leave in and what to leave out. Genes Dev 1994; 8: 1-8.

28 Tachibana M: Evidence to suggest that expression of MITF induces melanocyte differentiation and haploinsufficiency of MITF causes Waardenburg syndrome type2A. Pigment Cell Res 1997; 10: 25-33.

29 Nobukuni Y, Watanabe A, Takeda K, Skarka H, Tachibana M: Analyses of loss-of-function mutations of the MITF gene suggest that haploinsufficiency is a cause of Waardenburg syndrome type2A. Am J Hum Genet 1996; 59: 76-83.

30 Johnson D, Horlsey SW, Moloney DM et al: A comprehensive screen for TWIST mutations in patients with craniosynostosis identifies a new microdeletion syndrome of chromosome band 7p21.1. Am J Hum Genet 1998; 63: 1282-1293. 